\title{
Relative performance of the statistical learning network: An application of the price-quality relationship in the automobile
}

\author{
PierRe Desmet
}

\begin{abstract}
The design and topology of a neural network is still an important and difficult task. To solve the problems of topology posed by the introduction of connexionism, new approaches are proposed, and especially a combination of induction rules with a statistical estimation of the neuron coefficients for each layer. This research aims to compare an algorithm of this SLN approach with traditional methods (regression and classical BP neural networks) using the gradient method. Methods are put into application to determine the price-quality relationship of a complex product, the automobile, according to the hedonic price model. This application of the price-quality relationship to the English automobile market leads to the conclusion that the claimed superiority of this approach is unsubstantiated since, compared to the BP neural networks and even linear regression, the performance of the GMDH method is inferior.
\end{abstract}

\section{Introduction}

Connexionism constitutes a great advance for modelling in the sense that it allows for the representation of complexity using a network. The dimension most explored has been the consideration of non-linearity in relation to dependence, with the multi-layered Perceptron (MLP). This development has even led some authors to assert that it was about a dynamic change of direction creating a loss of interest in classical statistical methods (McGee and Kumar, 1992). So the era of "Data-mining" is opened up, promising the user transparent research, without hypothesis, of the most relevant indicators and the most suitable targets.

The price to pay for these advances achieved with artificial neuron networks is nevertheless heightened and bears on at least three dimensions:

(1) Estimation methods guaranteeing neither convergence, nor the obtention of the overall optimum; 
(2) The absence of rules for the topology of the network of which the configuration (the number of layers of neurons in each layer, the transfer functions in each layer) is left up to the user. The few empirical rules aimed at respecting a minimum number of degrees of freedom of estimated coefficients are not enough to guarantee optimality for the configuration;

(3) The loss of capacity for reflection on the results, of understanding the model, the analysis of the sources of the observed effects. This "black-box" approach leads to blind research by "Data-mining" in which the decision-maker loses the capacity for expert appraisal of his data and can resort only to simulation to measure the influence of the parameters that he considers important.

The tendency at present is in a combination of approaches, profiting from their complementarity, as with the genetic algorithm combination and MLP (Aurifeille, 1994; Khosla and Dillon, 1997). Researchers thus advocate the return of the classical statistical methods (regression) within the framework of connexionism (Lemke and Müller, 1995). Therefore, a new category of network has been proposed, the statistical learning neuron network (SLN), the acknowledged objective being to improve on traditional networks as regards the three aforementioned limits. They rest on the use of rules of induction to build a reiterative, polynomial and multi-layered neuron network where the coefficients are estimated by regression.

The objective of this research is to study the relative performance of this type of hybrid approach to a problem of which the empirical characteristics (low number of observations, strong colinearity of numerous variables, presence of specific variables). In spite of being a theoretically developed economic framework with hedonic prices, the answers given by statistical methodology are often still less than satisfactory. It revolves around the study of the price-quality relationship of a complex product, the automobile, a major management problem.

The rest of the article is organized in the following way: the first part presents the fundamental principles of the statistical learning network and the other methodologies used for comparison; the second part addresses the problem studied and its characteristics; and the third comments on the results obtained by the application of the various methods.

\section{Principles of statistical learning networks}

Proposed under a algorithmic form in 1967 by Ivakhmenko (Madala and Ivakmenko, 1994), the algorithm GMDH, i.e. Group Method of Data Handling, belongs to the class of statistical neuron networks with statistical learning (SLN). These methods share some characteristics with the classical neuron networks of (Farlow, 1984): first of all, connexionism as a representation of complex functions through a network of elementary functions (Morgan et al., 1997). Here the characteristic of the black box prevents the user, who is potentially unaware of the consequences of his choice, from carrying out all the topological choices in that it proposes only a very reduced number of options. The potential indecision, which results from the inappropriateness of the network consequently makes it impossible to take advantage of the information contained in the structure of the network or of all neuron coefficients. The SLN differs from the classical RNA in the fact 
that they are based on a statistical method, polynomial regression, for the determination of the coefficients.

While the classical GMDH supposes the a priori choice of the transfer function for all layers, the specificity of the recent approach, studied here, is in the automatic generation of the optimal partial model (Lemke, 1997). Recourse to the induction principle automatically determines the topology of a non-recurring network and it abolishes the necessity for a preliminary definition by the user of the network structure as is often the case with networks of the multi-layered Perceptron type. The objective of the hybridization method is to reduce over-learning and to find a structure having the lowest degree of effective non-linearity and assure a balance between complexity and performance.

In principle, this algorithm could thus be compared to a non-linear, multi-layered generalization by single-step regression. Optimization applies alternatively to the neuron coefficients and then to the transfer functions of the network structure.

The topology is determined in an iterative way, according to two principles: firstly, the auto-organization of the network, and, on the other hand, the validation of its performance by an externally adjusted qualitative criterion.

The method is described in the following stages (Lemke, 1997):

(a) Starting with a simple model: all the polynomial combinations of entry neuron pairs are created. The coefficients of the variable are estimated by regression on the exit neurons. The non-significant coefficients lead to the elimination of irrelevant variables.

$$
f\left(X_{i}, X_{j}\right)=a_{0}+a_{1} X_{i}+a_{2} X_{j}+a_{3} X_{i} \cdot X_{j}+a_{4} X_{i} \cdot X_{i}+a_{5} X_{j} \cdot X_{j}
$$

(b) Estimate of the contribution of the neuron to a validation sample. The cross-validatory method (Stone, 1974) is used. The programme determines the allocation of the observations between the evaluation and validation samples in a form which is clear to the user.

(c) Selection: like the genetic algorithms, several networks survive in parallel. The determination of survival is done according to a classification related to predictive power and a maximum number of surviving networks over a given period. At this stage another selection also takes place on the level of complexity of the network. If a new layer does not sufficiently improve the predictive quality, it is abandoned and the algorithm discontinued.

(d) Construction of the upper layer: the upper layer is constructed from the neuron survivors of the lower layer. Again, all the polynomial combinations of second degree neurons are constructed.

The combinatorial aspect of the partial models quickly leads to a great number of coefficients, which makes the program very consumptive in terms of calculation and storage space, even for relatively small databases compared to those in current use in marketing.

To estimate the relative performance of this algorithm, with its two options (linear and polynomial), two points of comparison are studied: a multi-layered perceptron and a classical regression which are opposed to SLN by the necessity to determine the topology of the system beforehand. However, with SLN (MLP), they share either the connexionist prejudice or the statistical evaluation of the coefficients (regression). 
These methods are going to be compared in regard to their capacity to resolve a complex and sizeable problem on the empirical plan: the evaluation of the value attributed by the market to the characteristics of a complex product.

\section{Problem: the price-quality relationship for a complex product}

To estimate the relative price for a complex product, the direct comparison of the prices for two alternatives is not enough because, first of all, these are not numerous and, on the other hand, they often have unique characteristics for the important features. So, before making the comparison, it is essential to bring back the prices to an identical base, in problematic terms "corrected prices" for quality differences.

This problem corresponds to an important need for a price policy on a market where the fixing of the price is strongly influenced by the competition. Within a multinational company it also corresponds to the price negotiation between central office and those responsible for subsidiaries who are confronted with specific local values for the features either because of the customers, or because of a local standard imposed by the leader. Nevertheless, the answers brought to this problem are still unsatisfactory in spite of a rigorously developed theoretical framework in economics, that of hedonic prices. This is due to the empirical characteristics of the problem which make the process of regression somewhat ill-adapted to data treatment, containing insufficient observations compared to the number of variables, often correlated and sometimes specific to each alternative. In moderating the consequences of these colinearities, the connexionist approach could be an interesting alternative solution.

\subsection{General model}

The fundamental hypothesis is that the price $(P)$ for a complex product is a function of its characteristics $(X): P=H\left(w_{j}\left(X_{j}\right)\right)$ with $w$ the function of evaluation of a characteristic and ' $H$ ' the function of the agregation of the characteristics (Eq. (1)). In the simple version of the model, the effects of interaction are not taken into account (i.e. the presence of a characteristic does not influence the value of another one) and the function $H$ is linear. If the hypothesis of an interaction is plausible, the buyer having a weaker price sensitivity than he accrues features, empirically this is often sacrificed as the decisionmaker searches a more stable and easily understandable model. A second simplification often considered intervenes for the function of evaluation $(w)$, it also being linear. For the most part this has no annoying consequences as regards the features which are present or absent, but could have for continuous variables such as engine rating. In this framework, the price (Eq. (2)) and the indication of the relative price (Eq. (3)) will then be spelt out simply, the price for product $i$ corrected for features present on $i$ and not on $j$, and conversely:

$$
\begin{gathered}
P_{i}=\Sigma_{j} w_{j} \cdot X_{j i}+w_{0 i} \\
\left.I_{i} l_{j}\right)=\left(P_{i}-\Sigma_{i j} w_{j} \cdot\left(X_{j i}-X_{j j}\right)\right) / P_{j}
\end{gathered}
$$




\subsection{Features}

The characteristics of complex products can be comparable or specific alternatively (Ladd and Zober, 1977). In the field of the automobile, it is, for example, about the presence or not of a braking system (ABS) or the constructor's trademark. The features can also be conceived in terms of physical characteristics and equipment or, indirectly, by characteristics of usage or service (insurance, the cost per $\mathrm{km}$, consumption, ....). In this research, only the objective accessible characteristics were studied along with those which could be considered as seen in the same way by every potential buyer.

Some features $(Y)$ don't have a given market value, while others $(X)$ can have a specific market value, even though this value is not directly accessible to the potential buyer because of the differences in pricing policy between the competitors. The determination of the function of product valorisation is therefore the most delicate step in the introduction of a model.

\subsection{The functions of valorisation}

Determination of the values a buyer accords to the characteristics is delicate because this value results from a combination of effects due to cost, economy of scale, demand, and competitive pressure.

- Cost approach: it consists of fixing the value of the feature from the company's production conditions, determined by expertise;

- Demand approach: stemming from the preferences expressed by a sample in an experimental framework, the joint analysis determines the perceived value of each feature (Levin and Johnson, 1984). Much used in practice, this model is however unwieldy to apply and of a predictive validity, requiring verification for each sample, considering the weak external validity;

- Competitive approach: starting from the principle that the market is at present in balance, the prices practised are close to optimal prices and reveal buyer preferences as much as production conditions (Rosen, 1974; Ohta and Griliches, 1972). Thus, observation and statistical analysis allow for the deduction of feature values, and this is the hedonic price approach (Boyer et al., 1984).

\subsection{Hedonic Prices}

The method of hedonic prices consists of statistically estimating the functions of valorisation $(w)$ of the features on a sample of products offered simultaneously on a market.

Several applications have already been carried out for various complex products such as housing or microcomputers, and especially the automobile (Cowling and Cubbin, 1972; Agarwal and Ratchford, 1980; Cubbin and Murfin, 1987; Goodman, 1989; Argea et al., 1994). However, this method has important limitations which renders its usage somewhat delicate on the empirical level. In effect, the estimated coefficients depend on the initial information contained in the studied sample and, in particular, they are subjected to the consequences of the colinearity between the features. Again, this colinearity 
is strong when the producers develop their marketing policies on sub-segments, as with the following paired factors 'motorization level $x$ equipment level' in the automobile sector. The practical consequence is that the coefficients obtained can run counter to basic common sense and lead to the total rejection of this approach by the user: being the case of a negative valuation of a feature or of an excessive valuation of equipment (high or low) in relation to its cost.

Hence, in the purely statistical approach, the joint approach is sometimes preferred which takes advantage of the information available with the price for the options. Taking the case of the joint external approach, the evaluation of the prices associated to the features $(Y)$ without a market value (such as engine rating) is only done after correcting the basic price for the presence / absence of the features $(X)$ having a market value.

$$
P_{i}^{*}=\left(P_{i}-\Sigma_{j} w_{j} \cdot X_{j i}\right)=\Sigma_{k} w_{k} \cdot Y_{k i}+w_{0 i} .
$$

The determination of the market value of the features is however subjected to the same difficulties because this value is subjective (determined "on the say of an expert") and because several estimates coexist. Furthermore the price for an optional feature can be considerably more than that of the same feature fitted as standard. The value of the reference used is then that of the option reduced by a certain percentage according to how often the feature is fitted as standard.

In this research, a joint internal model is proposed to improve the performance of the model and resolve the problem of the synthesis of market prices for the options, which can vary sometimes by a factor of 2 . The price for an option is added to that of the basic model to create a fictitious version.

The treatment of the colinearity of the features entails choices bearing heavy consequences: whether it be a question of the elimination of variables involved, which translates itself as an impoverishment of the model and by the non-consideration of variables considered important; or the use of a data reduction method of the type analysing only main components, which allows no further argument about decision-making features, but only about dissimilar features. Moderation of the consequences of colinearity, the specificity of the neuron networks of the Perceptron type, is a potentially important advantage for the hedonic price method. Furthermore RNA also avoids the expressed criticisms regarding regression which concentrates on the "average" relationship and imposes an arbitrary structure on relationships (Cubbin and Murfin, 1987).

\section{The empirical results}

\subsection{Data}

The application of the various methods for the determination of hedonic prices was done on data from the English automobile market in 1996. To limit the influence of the heterogenity of buyer preferences, only the small car sector (M1) was studied. The characteristics of the vehicles are available on data bases and diffused in the press from where they were collected (What car? Buyer guide, 1/96). 
- Variable objectives depicted by the bodywork, heavy equipment and safety, internal equipment and finish, have been noted and coded by auxiliary variables $(0 / 1)$. The motorization, a continuous variable, was expressed by two variables according to the fuel used (petrol/diesel). When several levels were proposed, the coding is cumulative and non-exclusive: for example, the electrically operated sun roof supposes a handoperated version. The list of the 48 variables appears in Appendix 1. The price is the officially noted price, before delivery expenses and negotiated prices.

- Selected vehicles: the models offered by the leading makes working on the English market were taken into account, with the exception of the special models $(4 \times 4)$. For every model, one auxiliary variable was created, making 15 in total, some makes offering several models (Fiat, Rover). The models are presented in their various versions which constitutes as many observations as there are samples: for example Volkswagen offers 14 versions of the Polo. The options proposed in the constructor's table were added to the least-well equipped basic versions for which they are offered, to establish a new alternative which is the price containing the value of the option.

The total number of observations amounted to 213 (131 versions, 82 options). The sample was divided into a working sample for the evaluation phase and a sample test composed of 21 vehicles selected at random from the versions offered by each constructor. For Perceptron, the working sample was separated into a learning sample (170) and a validation sample (22).

\subsection{Methods of application}

- SLN Network: The algorithm used was Knowledge-Miner. Of the three choices made only the first was explicit: the possibility of non-linear relationships, the minimal improvement of the solution so that the variables could be preserved simultaneously with the number of surviving models.

In the linear version, the following results were obtained: the algorithm retaining a tree of 6 levels, with two branches to every level, giving 55 coefficients in total. Considering the absence of polynomial terms, the structure simplified into a linear function with 12 coefficients which could be estimated by regression to obtain the classical statistical tests. In the non-linear version, 10 variables were retained for every 41 coefficients estimated. Compared to the linear version, some variables are common. The global results and the coefficients of retained variables are represented in the SLN column, tables 1 and 2 respectively.

- Multi-layered Perceptron: The topology not being defined, the empirical rule of the pyramid [the square root of the number of variables of the previous layer is used (Masters, 1994)], leading to 6 neurons for the first hidden layer and 2 for the second. To filter the colinearity, the first layer is linear and the second is Tanh (NN6L2T). Considering the large number of parameters to be determined (311), a network with the same transfer functions but a reduced number of neurons is also estimated ( 3 neurons and 1 neuron) with 153 parameters (NN3L1T). All the variables are standardized and the software used is SPSS's Neural Connection. 
- Linear regression: In the model, only significant variables were retained at a threshold of $15 \%$ and those for which colinearity was not perfect. Variables eliminated for the first reason were: the RDS audio system, the electrically-operated external rear mirrors, steering column and seat adjustment, electrically-operated sunroof, manual central locking, but also, more surprisingly, metallic paint although it is an option offered by all the selected constructors. Colinearity is perfect for the features which equip all the versions of a brand model, their valuation is thus embedded in the coefficient giving the specific value of the model. It concerns safety equipment (lateral struts and pretensioners), length of warranty and number of seats for the model. The regression applies to the working sample (192 observations) with the Ford Fiesta as a reference model. The statistical results lead to the overall acceptance of the model $\left(R^{2}=0.956, F=100.74\right)$.

\subsection{Comparison of the results}

Table 1 allows method performance to be compared from the point of view of overall efficiency. As the efficiency measures differed according to the methods (PESS and MAPE for SLN) or according to the samples used, one sole criterion was recalculated: the square root of the mean quadratic error brought back to the mean (RMS\%), as this indicator did not account for the number of degrees of freedom associated with the models, the number of estimated coefficients was recalled.

Overall, the adjustment is correct because the mean error is close to $5 \%$. The performance of the linear methods (regression and linear SLN) is lower on the sample test compared to the working sample. On the other hand, it is not the case for the more complex methods of topology: the results of the Perceptron seem relatively sound. However, the method hierarchy according to the performance is the same for the two samples. The mean residual deviation for the sample test (21 observations) shows the superiority of the most complex multi-layered Perceptron (NN6L2T). Linear regression has a credibly better performance than that of the simplified Perceptron (NN3L1T) which nevertheless contains nearly 5 times as many coefficients (153 against 36) which should have made it easier to adapt to the sample. On the other hand, the results of the SLN network are rather disappointing: in the linear version, this network is, by far, the least successful and the mean error is more than double that of regression. In the non-linear version, the performance improves but remains at a lower level than that of the other methods.

TABLE 1. Method performances (RMS\%).

\begin{tabular}{lccccc}
\hline Methods & Regression & \multicolumn{2}{c}{ Perceptron MLP } & \multicolumn{2}{c}{ SLN } \\
\hline Samples & REGLIN & NN3L1T & NN6L2T & Lin & Non-Lin \\
\hline (1) work & $4.13 \%$ & $4.49 \%$ & $3.33 \%$ & $8.37 \%$ & $6.79 \%$ \\
(2) test & $4.45 \%$ & $3.57 \%$ & $2.78 \%$ & $10.41 \%$ & $6.24 \%$ \\
Number of coefficients & 36 & 153 & 311 & 12 & 41 \\
\hline
\end{tabular}

As regards the face value of the coefficients obtained for every feature, only regression and linear SLN supply an explicit value for the parameter of the feature. To compare the 
results, a simulation was done by taking the example of a model to which an optional feature was added. Table 2 presents the results for some options as well as the list price and the quadratic mean for prices offered on the market which can be considered as a maxima value for the standard features fitted on the vehicle.

TABLE 2. Estimated value of features

(obtained by simulation).

\begin{tabular}{|c|c|c|c|c|c|c|c|}
\hline & $\begin{array}{c}\text { Linear } \\
\text { regression }\end{array}$ & $\begin{array}{c}\text { MLP } \\
\text { NN3L1T }\end{array}$ & $\begin{array}{c}\text { MLP } \\
\text { NN6L2T }\end{array}$ & $\begin{array}{l}\text { SLN- } \\
\text { Linear }\end{array}$ & $\begin{array}{c}\text { SLN- } \\
\text { Non Linear }\end{array}$ & $\begin{array}{l}\text { Price } \\
\text { option } \\
\text { brand }\end{array}$ & $\begin{array}{c}\text { Value } \\
\text { average } \\
\text { market }\end{array}$ \\
\hline Metallic Paint & 0 & 49 & -49 & 0 & 0 & 195 & 200 \\
\hline Driver'sAirbag & 244 & 541 & 375 & 683 & 0 & 260 & 263 \\
\hline Manual Sunroof & 305 & 476 & 339 & 395 & 590 & 240 & 299 \\
\hline RDS Radio & 0 & 821 & 481 & 0 & 0 & 240 & 180 \\
\hline $\begin{array}{l}\text { Electrically operated front } \\
\text { windows }\end{array}$ & 602 & -280 & 104 & 510 & 0 & 185 & 258 \\
\hline Alarm & 0 & 277 & -385 & 0 & 0 & 265 & 199 \\
\hline Automatic Gearbox & 601 & 405 & 317 & 0 & 0 & 680 & 818 \\
\hline Alloy Wheels & 618 & 390 & 19 & 0 & 0 & 350 & 336 \\
\hline ABS Braking System & 821 & 537 & 317 & 0 & 0 & 650 & 662 \\
\hline Leather Seats & 1846 & 1888 & 1614 & 2395 & 0 & 770 & 785 \\
\hline
\end{tabular}

Table 2 clearly shows the incapacity of SLN to take into account the most important features, which explains the weak overall performance obtained. SLN is supposed to be based on a balance and a controlled complexity, extracting all data information without falling into the pitfall of over- learning. However it seems that the network stops short of a sufficient level of complexity. All the coefficients considered for linear regression are positive, which is not the case for the neuron networks. But it is difficult to accept a negative evaluation for the features in the empirical plan. Finally, it is noticeable that some coefficients are overvalued by the various methods (e.g. fitted leather seats), which can be explained by the specificity of this feature on a given range level and hence by its underlying colinearity. In summary, this detailed analysis show that only regression gives an acceptable result whether at the level of overall error or at the level of specific evaluation of the features.

\section{Conclusion}

To resolve the problems linked with the use of the neuron networks, such as the determination of the topology and the valuation of the coefficients, while retaining the advantages of connexionism, some new approaches are proposed. Here, the statistical learning neuron network would propose resorting to induction to determine the optimal topology of the network, and to regression for the evaluation of the neuron coefficients.

A comparative analysis was carried out on a delicate and major management problem for which very little data was either available or correlated, and having different types of features. The results are rather disappointing for those contributing to this new type of network using a hybrid step. In effect, the overall performance of the SLN networks, 
measured in terms of residual error, is much lower than that of the multi-layered Perceptron and, furthermore, lower than that of classical regression. The detailed analysis of the obtained coefficients either directly for regression, or by simulation for the other methods, shows that the Perceptron type networks can also give a negative valuation of some fittings. The modification proposed to the method of classical hedonic prices, by the consideration of the options under the form of supplementary versions, produces equally acceptable results as regards regression for overall adjustment as for the valorisation of the fittings. This method thus establishes a new reference point with regard to the possibility of comparing the results of a methodology combining a network structure and parameters determined by a genetic algorithm, which would allow control of the constraint of non-negative coefficients.

\section{Appendix}

\section{A. List of features used.}

Number of seats (3/4),

Number of doors (3/5),

Coupe (normal/cabriolet),

Sun-roof, manuall or electrical,

Metallic paint,

Alloy wheels,

Power rating, Petrol/Diesel,

Motorisation $16 \mathrm{~V}$,

Injection,

Electrically-operated front windows,

Electrically-operated rear mirrors,

Folding rear seats, with split,

Leather seats,

Adjustable seats,
Power steering,

ABS braking system,

Automatic gearbox,

Air conditioned,

Driver's Airbag, Passenger's Airbag,

Lateral Struts,

Pretensioned Safety Belt,

Remote central locking,

Anti-theft device Immobilisation of Vehicle,

Alarm,

Adjustable steering column,

Cassette player, RDS Radio,

CD player,

Warranty (12 or 36 months)

B. Liste of makes (and models)

Citroën (AX), Fiat (Cinquecento, Uno, Punto), Ford (Fiesta), Nissan (Micra), Peugeot (106), Renault (Clio), Rover (R111, R114, R115), Seat (Ibiza), Suzuki (Swift), OpelVauxhall (Corsa), Volkswagen (Polo)

\section{References}

Agarwal M., Ratchford B. (1980) Estimating Demand Functions For Product Characteristics: The Case of Automobile, J Consumer Res. 7, December, pp. 249-261.

Argea N., Hsiao C., Taylor G. (1994) Estimating Consumer Preference Using Market Data: An Application to US Automobile Demand, J. Appl. Econometrics 9, pp. 1-18.

Aurifeille J.-M. (1994) Réseaux de neurones et Analyse des données en Marketing: Intérêts, Limites et Perspectives", Proceedings Première Rencontre ACSEG, Poitiers, France, pp. 3-25. 
Boyer A., Palda K., Ratchford B. (1984) The Hedonic Approach to Price-Quality Relationship and its Potential Application in Marketing, Research in Marketing 7, pp. 1-40.

Cowling K., Cubbin J. (1972) Hedonic Price Indexes for UK cars. Economic J. 82, pp. 963-978.

Cubbin J., Murfin A. (1987) Regression Analysis versus Linear Programming in the Analysis of Price-Quality Relationships: an Application to the Determination of Market Share. Oxford Bulletin of Economics and Statistics 49, 4, pp. 385-399.

Farlow S.J. (1984) Self Organizing Methods in Modeling: GMDH Type Algorithm, Marcel Dekker, New York.

Goodman A.C. (1989) Willingness to Pay for Car Efficiency: The Hedonic Price Approach, J. Transport Economics and Policy 17, pp. 247-266.

Khosla R., Dillon T. (1997) Engineering Intelligent Hybrid Multi-agent Systems, Kluwer Academic Publishers, London.

Ladd G., Zober M. (1977) Model of Consumer Reaction to Product Characteristics, J. Consumer Res. 4, pp. 89-101.

Lemke F. (1997) Knowledge Extraction from Data using Self-organizing Modeling Technologies, SEAM' 97 Conference (www.scriptsoftware.com).

Lemke F., Müller J. (1997) Self Organizing Datamining for a Portfolio Tracking System. J. Computational Intelligence in Finance 5, 3, pp. 18-24.

Levin I., Johnson R. (1984) Estimating Price-Quality Trade-offs using Comparative Judgments. J. Consumer Research 11, June, pp. 593-600.

Madala H.R., Ivakhnenko A.G. (1994) Inductive Learning Algorithms for Complex System Modelling, CRC Press Inc, Ann Arbor.

Masters T. (1994) Pratical Neural Network Recipes in $C^{++}$, Academic Press, NY.

McGee V., Kumar A. (1992) Regression and Neural Networks: Time for a Change?, W.P. Amos Tuck School of Business Administration, Dartmouth College, Hanover, NH.

Morgan P., Curry B., Beynon M. (1997) Comparing Neural Network Approximations for Different Funstional Forms, Proceedings Quatrième Rencontre Internationale ACSEG, Tours, France, pp. 112-126.

Ohta M., Griliches Z. (1972) Makes and Depreciation in the US passenger Car Market: An Application of the Hedonic Hypotheses to the Construction of Price Indexes and the Study of Market Structure, Mimeographed, Harvard University.

Rosen S. (1974) Hedonic Prices and Implicit Markets : Product Differentiation in Pure Competition. J. Political Economy 82, pp. 34-55.

Stone M. (1974) Cross-validatory Choice and assessment of statistical predictions. J. The Royal Statistical Society B36, pp. 111-147. 